\title{
Exploiting Downstream Mobility to Achieve Fast Upstream Message Propagation in Vehicular Ad Hoc Networks*
}

\author{
A. Agarwal, D. Starobinski, and T.D.C. Little \\ Department of Electrical and Computer Engineering \\ Boston University, Boston, Massachusetts \\ $\{$ ashisha,staro,tdcl\}@bu.edu
}

MCL Technical Report No. 05-03-2007

\begin{abstract}
Vehicular ad-hoc networks (VANETs) promise to enable many novel applications in transportation systems including accident avoidance, congestion sensing, traffic metering, and general in-car information services. Yet implementing multi-hop vehicular communications is highly challenging due to the highly time-varying nature of vehicles. In addition to node mobility and the impediments of wireless communication, a network comprised of moving vehicles is a dynamic system that can be fragmented into many individual components of disparate connectivity. In this paper we describe and analyze a routing scheme that exploits this dynamic connectivity for the purpose of message propagation of attributed (or labelled) data in a fragmented VANET. Our analysis provides upper and lower bounds on message propagation rate as a function of the traffic density, vehicle speed, and radio range; and sheds light into the role played by each of these network parameters. An important insight from our analysis is that vehicle mobility in the opposite traffic direction can be used to achieve substantial gains in message propagation rates. Our analytical findings are supported by extensive simulations. The simulation results indicate that under certain traffic conditions an increase in vehicle mobility results in an order-of-magnitude increase in message propagation rate.
\end{abstract}

\footnotetext{
*In Proc. Mobile Networking for Vehicular Environments 2007, Infocom 2007, Anchorage, AK, May 2007. This work was supported by the NSF under grant Nos. CNS-0435353, CNS-0132802, CNS-0240333, and CNS-0435312. Any opinions, findings, and conclusions or recommendations expressed in this material are those of the author(s) and do not necessarily reflect the views of the National Science Foundation.
} 


\section{Introduction}

Vehicular ad hoc networks (VANETs) promise to enable a wide range of novel applications in transportation systems. Potential applications include point-to-point communication (e.g., mobile VoIP), accident avoidance messaging, congestion sensing, traffic metering, and general information services (e.g., Internet access) [1]. Supporting future large-scale vehicular networks is expected to require a combination of fixed roadside infrastructure and mobile in-vehicle technologies. Enabling vehicular communication with rapid and autonomous behavior suitable for safety applications will clearly require the use of multihop ad hoc network concepts.

Data exchange and messaging requirements for applications are diverse. Safety applications are typically confined to short ranges with requirements of low latencies and small payloads. Conversely, traffic information systems are designed to collect and interpret data originating from many vehicles in relatively large areas. Messaging is distributed over a greater region with tolerance for delays in data delivery. Similarly, applications such as Internet and in-vehicle entertainment systems are expected to require high asymmetric data rates. In this context we are interested in vehicular traffic behavior and its impact on these general types of applications.

Although they are a class of mobile ad hoc network (MANETs), VANETs have unique behaviors. Vehicles can have very predictable movement that is (usually) limited to roadways. Roadway information is often available from positioning systems and map-based technologies such as GPS. Traffic density can vary significantly based on time-of-day and day-of-week. With respect to connectivity, density plays a key role in enabling multihop communication. Owing to the high, path-like mobility rate and varying traffic density observed, a VANET is characterized by timevarying topology and connectivity [2]. Hence, the topology of VANET is usually partitioned, and is better described by a fragmentation and fusion process. Fragmentation and fusion lead to many problems with conventional MANET routing schemes, especially proactive ones. While existing routing protocols have been investigated for applications in this domain, few have tried to use the opportunistic connectivity provided by mobile vehicular nodes.

Our work, as described in this paper, is towards characterizing this fragmentation under mobility and its impact on message propagation. We introduce an analytical model to evaluate the behavior of this propagation over moving vehicles under a delay tolerance assumption. We describe a routing scheme from existing work [3] that exploits the time-varying connectivity for message propagation in a fragmented network. The work demonstrates that vehicle mobility can be exploited to achieve substantial gains in the message propagation rate even under conditions of fragmentation. Our analysis considers varying traffic density and provides bounds on message propagation rates as a function of vehicular speed, radio range, and traffic density.

The remainder of the paper is organized as follows: Section II describes related work. In Section III we describe the vehicular networking scenario, assumptions, and the base routing scheme. A model and corresponding analysis of behavior of message propagation is presented in Section IV followed by simulation results in Section V. Section VI concludes the paper with a summary and discussion of the results. 


\section{Related Work}

The IEEE WAVE (Wireless Access for Vehicular Environments) is a group formed to develop standards and protocols for inter-vehicle communication using the dedicated short-range communications band at $5.9 \mathrm{GHz}$ [4]. Their goal is to develop and standardize mechanisms to allow vehicles to exchange information for the purpose of safety enhancement. Vehicle-to-vehicle communications has been explored in projects called FleetNet and CarTALK [5]. The VANET approach used in CarTALK is characterized by the use of map information, GPS coordinates, and spatially-aware routing. In the FleetNet project, the authors explore the prospect of using roadside gateways to deal with the sparse traffic scenario. By contacting gateways, clusters of vehicles associated with a local FleetNet routing group are able to bridge gaps due to network fragmentation. With a goal of providing additional services including connection-oriented traffic, this approach requires additional mechanisms and the deployment of typically costly infrastructure. Such schemes assume complete connectivity of the network or require specific geo-spatial mapping information. Compared to our work, we instead focus on cases in which data can tolerate some delay as would be expected when fragmentation is present. We use a model characterized by constant network fragmentation due to transiting blocks of vehicles. We also use a routing based on labeled data, which offers some advantages in information dissemination.

Delay tolerant networking (DTN) and custody transfer [6] are concepts that we apply here in conjunction with the use of labeled data. DTN is essentially a store-and-forward scheme that assumes a partitioned network. The concept assumes that messages are stored in the memory of a node and forwarded whenever connectivity to the next hop is available. As the messaging is connectionless, accounting for acknowledgment of message delivery is a difficult task. The custody transfer concept permits an exchange of responsibility for forwarding messages from one node to the next in forward progress towards a destination. Data or nodes are assumed not to be lost in the network.

Wu et al. have proposed an analytical model to represent a highway-vehicle scenario [7]. However, while they consider a partitioned network, they do not consider the use of opposing traffic to propagate data. Rather, they investigate speed differential between vehicles traveling in the same direction to bridge gaps and propagate data. In our model, we demonstrate that the transient connectivity offered by opposing traffic can provide a substantial improvement in message propagation rate.

\section{VANET Scenario and Routing Scheme}

We concentrate on information propagation on a roadway without infrastructure; one in which multiple vehicles transit in both directions. The roadway is modeled as rectilinear as illustrated in Figure 1. We also assume that packet radio is tolerant to local variations in directionality and curvature of the roadway. Vehicles are equipped with sensing, communication, and computing capabilities so that they form nodes of an infrastructure-less ad hoc network. Vehicles are assumed to travel at a constant velocity. 
A time-series of snapshots demonstrates that vehicles are grouped in small disconnected clusters. This has been observed in vehicular traffic data $[8,2]$. By assuming a fixed packet radio transmission radius, these data indicate a corresponding clustering and fragmentation of vehicles on a unidirectional roadway. Formation of end-to-end paths is difficult, if not impossible, especially in low vehicular density scenarios. In networking terminology, the connectivity graph formed by vehicles can be described as a partition yielding multiple disconnected subnets. If nodes traveling in opposing direction are used in path formation, the resulting paths are short-lived, leading to considerable overheads in dynamic path formation and route maintenance. Thus, MANET routing protocols which rely on such strategies are a poor solution.

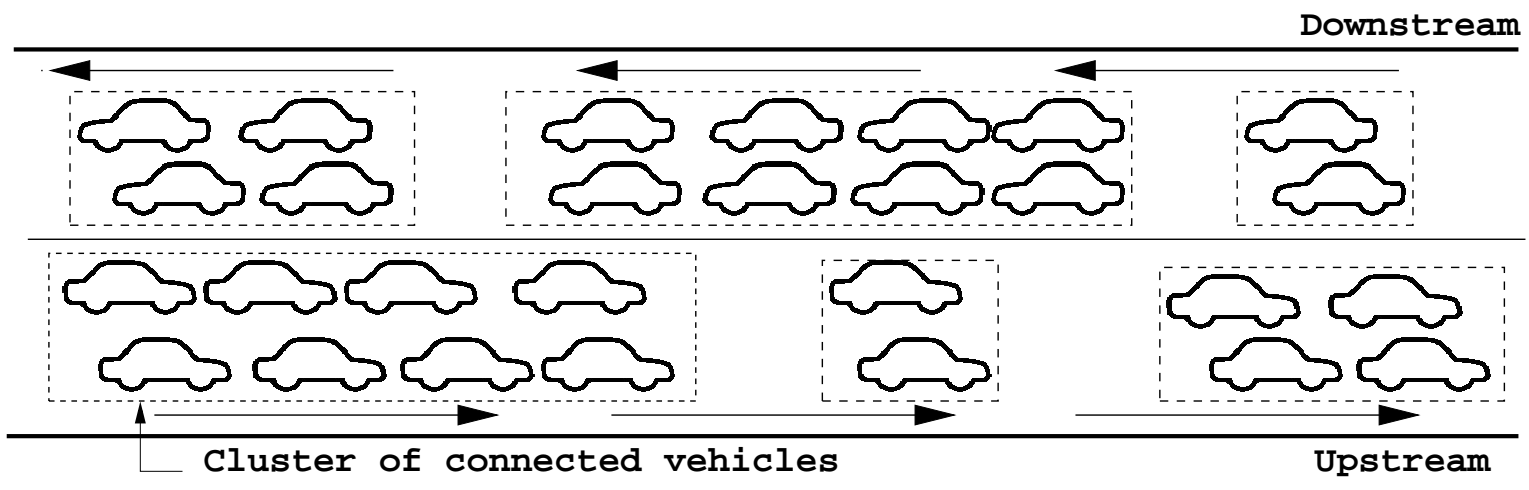

Figure 1: Illustration of the fragmented highway scenario.

We consider a routing scheme of the form proposed in reference [3]. This scheme is based on message labeling using descriptive tags supporting the message propagation goal. For example, with the availability of location and map information from GPS devices in vehicles, messages can be attributed with source and destination locations or regions. These tags, or attributes, work in conjunction with a message time-to-live (TTL), a function of time and area, that provides control over the extent of dissemination away from a traffic incident. To bridge traffic clusters (fragments) a custody transfer scheme is adopted from the delay tolerant networking [6]. Based on attributes such as location, heading, time, etc., this scheme achieves directional propagation of messages in a network of moving vehicles.

An end-to-end path from source to destination may not exist when fragmentation occurs. To route messages, a path from source to destination is not formed a priori; rather, the protocol uses opportunistic connectivity between nodes and traffic flow in both directions. Messages and vehicles travel either upstream or downstream relative to their counterparts. Upstream messages are forwarded by vehicles traveling upstream by exploiting commonality of direction. When a partition in upstream traffic is encountered, messages can be forwarded via downstream traffic to exploit possible connectivity there. This downstream traffic is, in some cases, sufficient to bridge the partition. With the help of attributed data, the protocol is able to maintain the directional propagation of data even with transient connectivity. Thus, this scheme exploits a highly time-varying connectivity in an opportunistic way to bridge partitions. The directional propagation achieved by this mechanism is illustrated in Figure 2.

The scheme performs comparably to similar map-based approaches based on path formation 


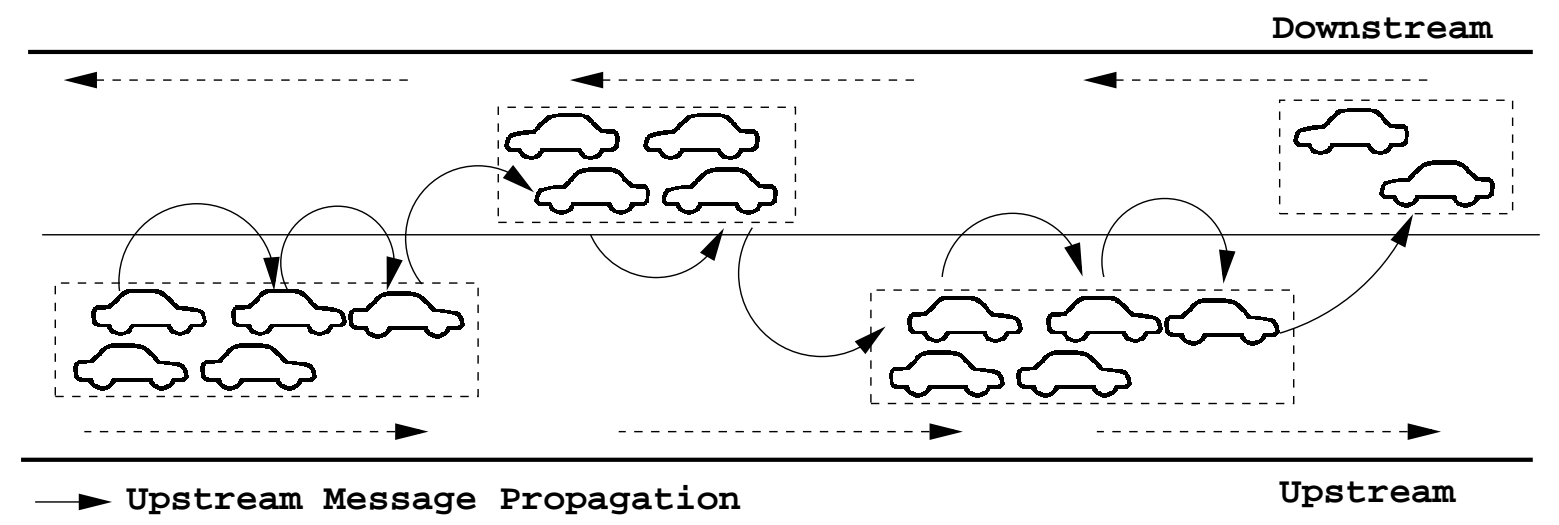

Figure 2: Illustration of directional propagation of data in a VANET.

strategies in a fully connected scenario. An interesting observation is the performance of the scheme in a partitioned environment with time varying connectivity. Whereas, certain approaches would fail as result of non-existent end-to-end paths, this scheme is able to exploit the transient connectivity offered by vehicular traffic traveling in opposite direction. Thus, our main focus in this paper is accurately characterize this behavior and performance.

\section{VANET Model and Analysis}

Consider a highway scenario as described in Section III. The model is a simplified linear arrangement of vehicles located randomly along the line. Vehicles are mutually separated by distance $x$ (Fig. 3). We define a radius of connectivity $R$, that determines which vehicles are connected. That is, they are connected if $x \leq R$. Under dense traffic conditions, there are multiple vehicles on the road and these permit multihop connectivity. Under sparse traffic conditions, the vehicles are largely disconnected. We assume that vehicle size (length) to be negligible in terms of its impact on connectivity.

We describe a scenario wherein the upstream vehicles are separated by a fixed distance $d>R$, such that they are disconnected (outside of radio range). While for downstream traffic, (vehicles traveling in the opposite direction) the inter-vehicular distance is distributed as an exponentially random variable with parameter $\lambda$. Such an arrangement is chosen to specifically model the case in which vehicular traffic has partitions. Our objective is to characterize the behavior of time-varying connectivity.

The length of the roadway for downstream traffic is divided into cells each of size $l$ (Fig. 3). We consider two bounds for the cell size; $R$, an upper bound, and $R / 2$, a lower bound. When the cell size is $R$, the distance between the vehicles can be greater than $R$ when the vehicles are located at the two ends of a cell. Thus, with cell size $R$, the nodes are not necessarily connected. However, there are possible arrangements where the nodes could be connected as illustrated in Figure 4. At the lower bound $R / 2$, we ensure that the nodes are always connected. However, there are cases in which there are more nodes than are required to achieve connectivity. Thus, the upper bound is 


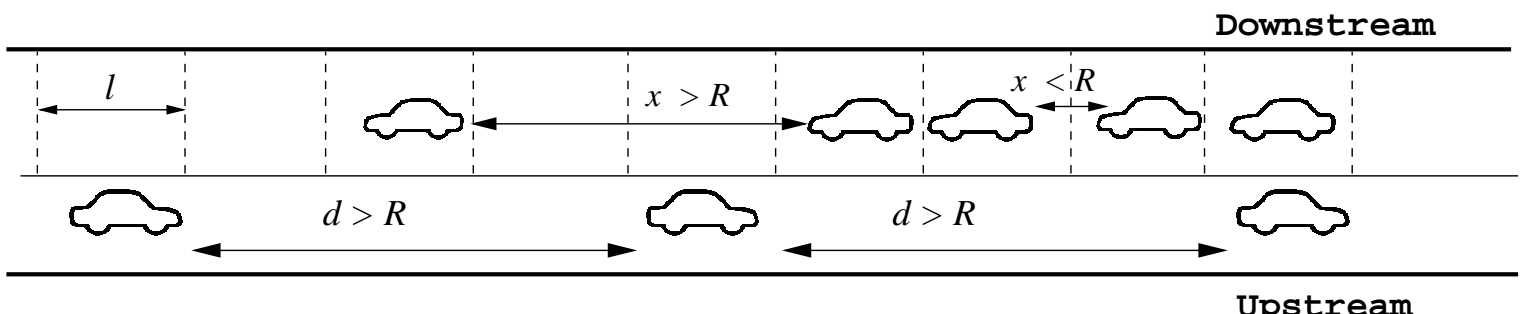

Figure 3: Illustration of the highway model.

a necessary but not sufficient condition. Whereas, the lower bound is a sufficient but not always necessary condition. The two cases are illustrated in Figure 4.

(a) Upper bound

Downstream

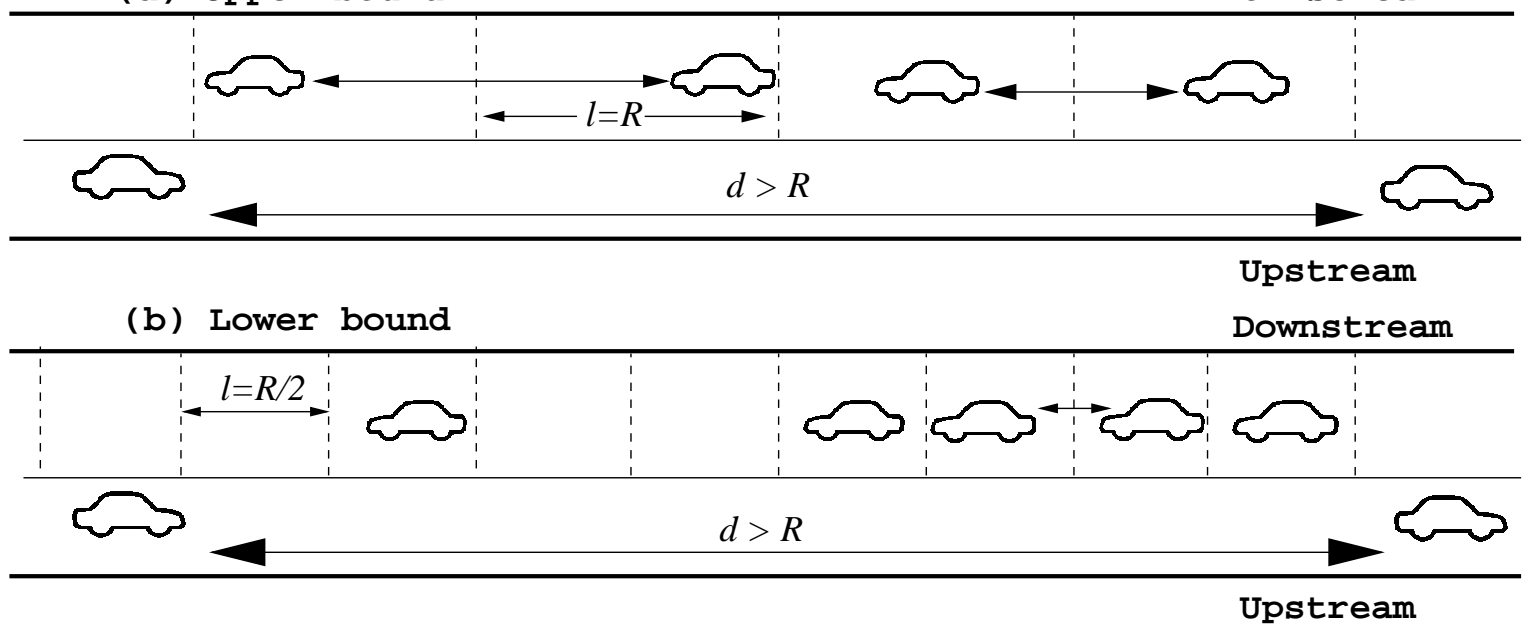

Figure 4: Illustration of upper and lower bounds.

Traffic along the downstream direction is assumed to be exponentially distributed. The arrival process is chosen to be exponential to achieve the independence property in the analysis. The probability that a cell of length $l$ is occupied is $p$. With the assumption of exponentially distributed inter-vehicle distance along downstream traffic, the probability that a cell is occupied is given by $p_{u p}=\left(1-e^{-\lambda R}\right)$ and $p_{l o w}=\left(1-e^{-\lambda R / 2}\right)$, where up and low denote upper and lower bounds. Correspondingly, the number of cells in the gap $d$ are $k_{u p}=\lfloor d / R\rfloor$ and $k_{\text {low }}=\lfloor 2 d / R\rfloor$. For the sake of brevity, we will consider $p$ and $k$ for analysis and substitute for upper and lower bounds.

Vehicles are assumed to move at a speed of $v \mathrm{~m} / \mathrm{s}$. We define a message propagation speed $v_{\text {radio }}$. This speed is determined by the characteristics of the packet radio physical layer and the multihop network later, and is approximated by the maximum distance propagated, including intermediate hops, per second. For simplicity, we initially assume that $v<<v_{\text {radio }}$, that is, messages travel much faster than vehicles. We relax this assumption towards the end of the analysis. One metric to estimate the performance of the network is the distance covered by the message in the given topology. As described earlier, there are alternating periods of multihop connectivity and disconnection. We call these Phase 1 and Phase 2, respectively. In Phase 1, when there is multihop 
connectivity available the messages propagate at the speed of the radio over connected vehicles. While in Phase 2, when vehicles are disconnected, the messages propagate at vehicle speed waiting for additional connectivity. We define $v_{\text {eff }}$ as the effective propagation rate which is a function of the time and distance in the two phases.

Theorem 4.1 The effective propagation rate $v_{\text {eff }}$ lies between the bounds: $v_{l o w} \leq v_{\text {eff }} \leq v_{\text {up }}$; where $v_{u p}$ is an upper bound on the propagation rate, given by:

$$
v_{u p}=\frac{E\left[D_{1}\right]_{u p}+E\left[D_{2}\right]_{u p}}{E\left[D_{1}\right]_{u p} / v_{\text {radio }}+\left(E\left[D_{2}\right]_{u p}-d\right) / v+d / v_{\text {radio }}}
$$

and $v_{\text {low }}$ is a lower bound given by:

$$
v_{\text {low }}=\frac{E\left[D_{1}\right]_{\text {low }}+E\left[D_{2}\right]_{\text {low }}}{E\left[D_{1}\right]_{\text {low }} / v_{\text {radio }}+\left(E\left[D_{2}\right]_{\text {low }}-d\right) / v+d / v_{\text {radio }}}
$$

where $E\left[D_{1}\right]_{\text {low }}, E\left[D_{2}\right]_{\text {low }}, E\left[D_{1}\right]_{\text {up }}$ and $E\left[D_{2}\right]_{\text {up }}$ are the lower and upper bound values of expected distances covered in Phases 1 and 2 respectively. $E\left[D_{1}\right]=\frac{d p^{k}}{1-p^{k}}$ and $E\left[D_{2}\right]=\frac{\left(1-p^{k}\right)}{(1-p) p^{k}} \frac{l}{2}+d$, where $p$ is the probability a cell of size $l$ is occupied and $k$ is the number of cells in the gap $d$.

Proof: In Phase 1, the nodes are connected by multiple hops and the message is able to propagate over connected vehicles. For a multihop path to the next hop upstream, each downstream cell in the gap $d$ must have at least one vehicle. The probability of such an event is given by $p^{k}$, where $p$ is probability that a cell is occupied while $k$ is the number of cells. The probability that a distance of $D_{1}$ is covered is expressed as $\operatorname{Pr}\left\{D_{1}=n d\right\}=\left(p^{k}\right)^{n}\left(1-p^{k}\right)$. We compute the expected value of distance $D_{1}$ :

$$
E\left[D_{1}\right]=\frac{d p^{k}}{1-p^{k}}
$$

In Phase 2, conversely, one or more cells in the downstream traffic is not occupied by a vehicle, and thus, there is an absence of multihop connectivity. The message are cached until an event in which all $k$ cells in the gap have at least one vehicle. While waiting for this event, the vehicle and the message traverse a number of cells at a speed of $v \mathrm{~m} / \mathrm{s}$. The number of cells traversed until the desired event is analogous to the number of trials until a particular sequence is seen, described as pattern matching in classical probability theory [9]. The pattern matching problem describes the task to compute the expected number of trials until $k$ consecutive successes. It is analogous to our problem, as we try to find the number of cells traversed by the vehicle, until it finds all cells along the downstream traffic to be occupied by one or more vehicles. Equivalently, the number of trials until the inter-vehicle distance between all cells is less than $R$, for the gap $d$. Under this condition, connectivity to the next hop is achieved, resulting in successful message propagation. The expectation above accounts for the cases in which one or more cells in the gap $d$ is not occupied by a vehicle.

¿From known results on pattern matching [9], the expected number of cells, $N$, traversed until desired event is: $\sum_{i=1}^{k} 1 / p^{i}$ or equivalently, $E[N]=\frac{\left(1-p^{k}\right)}{(1-p) p^{k}}$ 
Each cell is of size $l$, thus, we can compute the distance until the desired connectivity. The message covers a distance of $E[N]-k$ cells with the vehicle at speed $v$. Once the connectivity is available, it traverses the $k$ cells at speed $v_{\text {radio }}$. Since traffic in both directions is moving at the same speed, the distance covered by message until connectivity is adjusted by a factor of $1 / 2$. Thus, the distance covered by message in Phase 2 is given by:

$$
E\left[D_{2}\right]=\left(\frac{\left(1-p^{k}\right)}{(1-p) p^{k}}-k\right)\left(\frac{l}{2}\right)+d
$$

Applying upper and lower bounds to equations (1) and (2):

$$
\begin{aligned}
& E\left[D_{1}\right]_{u p}=\frac{d\left(1-e^{-\lambda R}\lfloor\lfloor d / R\rfloor\right.}{\left(1-\left(1-e^{-\lambda R)\lfloor d / R\rfloor}\right)\right.} \\
& E\left[D_{1}\right]_{l o w}=\frac{d\left(1-e^{-\lambda R / 2}\right)^{\lfloor 2 d / R\rfloor}}{\left(1-\left(1-e^{-\lambda R / 2}\right)^{\lfloor 2 d / R\rfloor}\right)} \\
& E\left[D_{2}\right]_{u p}=\left(\frac{\left(1-\left(1-e^{-\lambda R}\right)^{\lfloor d / R\rfloor}\right)}{e^{-\lambda R}\left(\left(1-e^{-\lambda R}\right)^{\lfloor/ R\rfloor}\right)}-\frac{d}{R}\right) \frac{R}{2}+d \\
& E\left[D_{2}\right]_{\text {low }}=\left(\frac{1-\left(1-e^{-\lambda R / 2}\right)^{\lfloor 2 d / R\rfloor}}{e^{-\lambda R / 2}\left(\left(1-e^{-\lambda R / 2}\right)^{\lfloor 2 d / R\rfloor}\right)}-\frac{2 d}{R}\right) \frac{R}{4}+\frac{d}{2}+d
\end{aligned}
$$

For the lower bound, we include an additional compensation of $d / 2$, which is the distance, in the worst case, a message must cover when it alternates from Phase 1 to Phase 2 (transition from Phase 1 to Phase 2 implies that one of the cells must be empty; pattern matching trials can start only after that empty cell.). Substituting the derived expressions, the effective propagation rates for the upper and lower bounds can be expressed as:

$$
\begin{gathered}
v_{\text {up }}=\frac{E\left[D_{1}\right]_{\text {up }}+E\left[D_{2}\right]_{\text {up }}}{E\left[D_{1}\right]_{\text {up }} / v_{\text {radio }}+\left(E\left[D_{2}\right]_{\text {up }}-d\right) / v+d / v_{\text {radio }}} \\
v_{\text {low }}=\frac{E\left[D_{1}\right]_{\text {low }}+E\left[D_{2}\right]_{\text {low }}}{E\left[D_{1}\right]_{\text {low }} / v_{\text {radio }}+\left(E\left[D_{2}\right]_{\text {low }}-d\right) / v+d / v_{\text {radio }}}
\end{gathered}
$$

Finally, we relax the assumption $v<<v_{\text {radio. }}$. Suppose the distance between vehicles in the upstream traffic is $d$. All vehicles, both along upstream and downstream directions, move at speed $v$. The effective gap between upstream nodes becomes $d^{\prime}=d+v t$, where $t$ is the time taken to cover the distance. The messages traverse the same distance downstream at speed $v_{\text {radio }}-v$, which implies: $d^{\prime}=t\left(v_{\text {radio }}-v\right)$. Thus, the effective gap $d^{\prime}$ is expressed as:

$$
d^{\prime}=d+\frac{d v}{v_{\text {radio }}-2 v}
$$

The replacement of $d$ by $d^{\prime}$ can be applied to all the derived upper and lower bound formulas.

\section{Simulation Results}

We evaluate the analytical model against simulation for varying traffic density. For the simulation, we choose parameters of message propagation speed as $v_{\text {radio }}=1000 \mathrm{~m} / \mathrm{s}$ and inter-vehicular distance $d=300 \mathrm{~m}$. The radio range is $R=125 \mathrm{~m}$ and vehicle speed is assumed to be $v=20 \mathrm{~m} / \mathrm{s}$. 


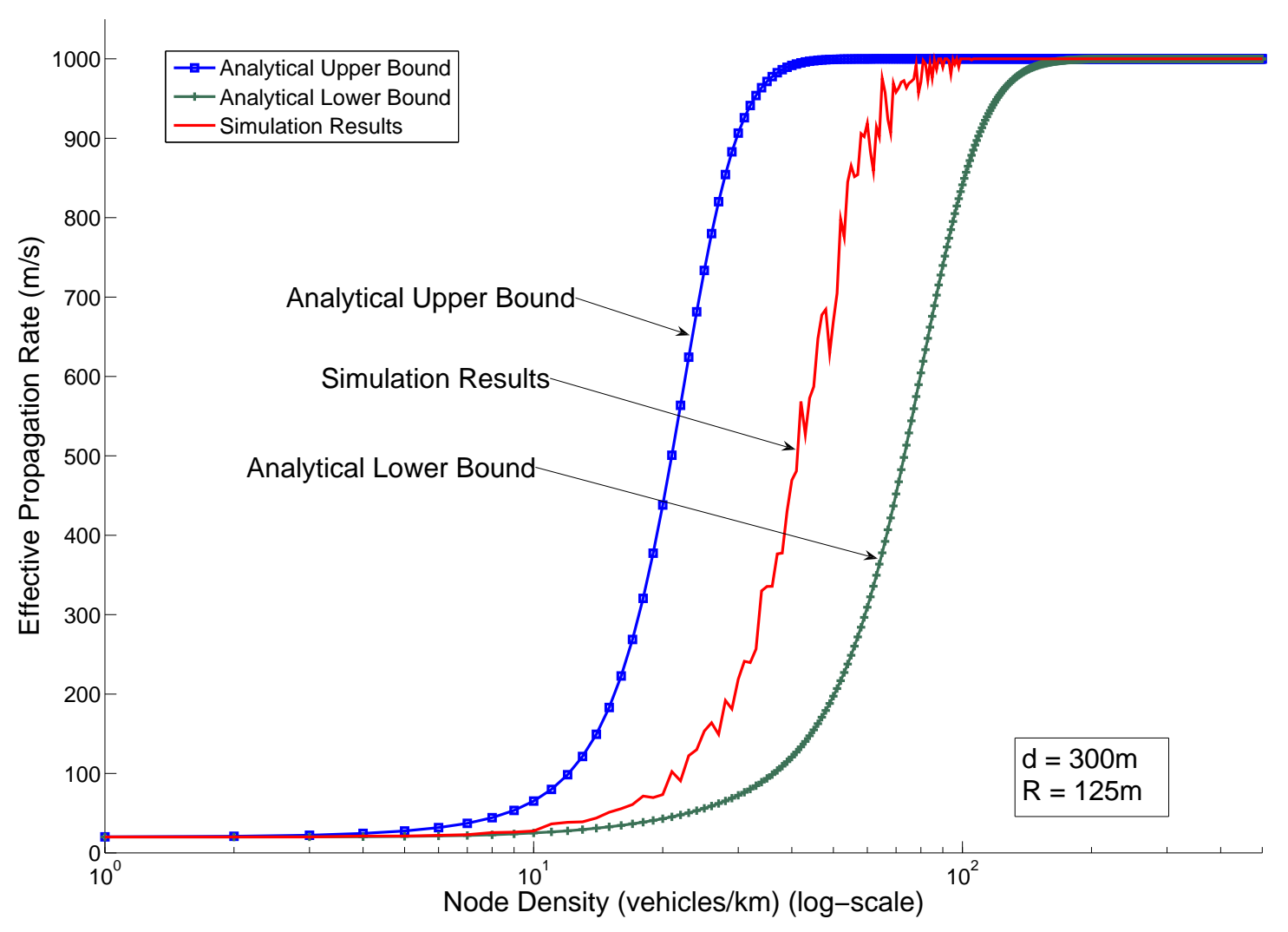

Figure 5: Comparison of Analytical Model and Simulation Results

We consider the downstream traffic density over a range of 1 vehicles $/ \mathrm{km}$ to 500 vehicles $/ \mathrm{km}$ to cover the low, intermediate and high traffic density scenarios. The simulation results are obtained from a model that generates traffic with fixed traffic in the upstream direction and inter-vehicle distance that is exponentially distributed in the downstream direction. The propagation rates achieved for the routing scheme described in Section IV are plotted in Figure 5. The simulation results are repeated and averaged to account for the random arrival process. ¿From the graph, it is apparent that when there is sparse traffic, messages traverse the system at a speed $v$ corresponding to vehicular motion due to the lack of multihop connectivity. In intermediate traffic, as density increases, the messages wait less often for connectivity and the rate of propagation increases. In high traffic, the network is mostly fully-connected and propagation is achieved at a rate equal to $v_{\text {radio }}$.

These results also show that the propagation rate lies predominantly in two regimes. At one extreme is the low density case; the network is disconnected and the propagation rate is minimal. The other extreme is the high density scenario which yields a fully-connected network and maximal propagation rate. More interesting behavior is observed in the intermediate density in which opportunistic connections are used to achieve improved message propagation. As shown in Figure 6 , the densities at which the various regimes are observed are dependent upon the vehicular speed. The graph shows that at a slower speed of $v=0.1 \mathrm{~m} / \mathrm{s}$, a higher density of vehicular nodes is required for achieving the same performance as nodes travelling at $v=20 \mathrm{~m} / \mathrm{s}$. It is evident from equations (3) and (4), the gain in message propagation rate is linear with vehicular speed $v$ for low 


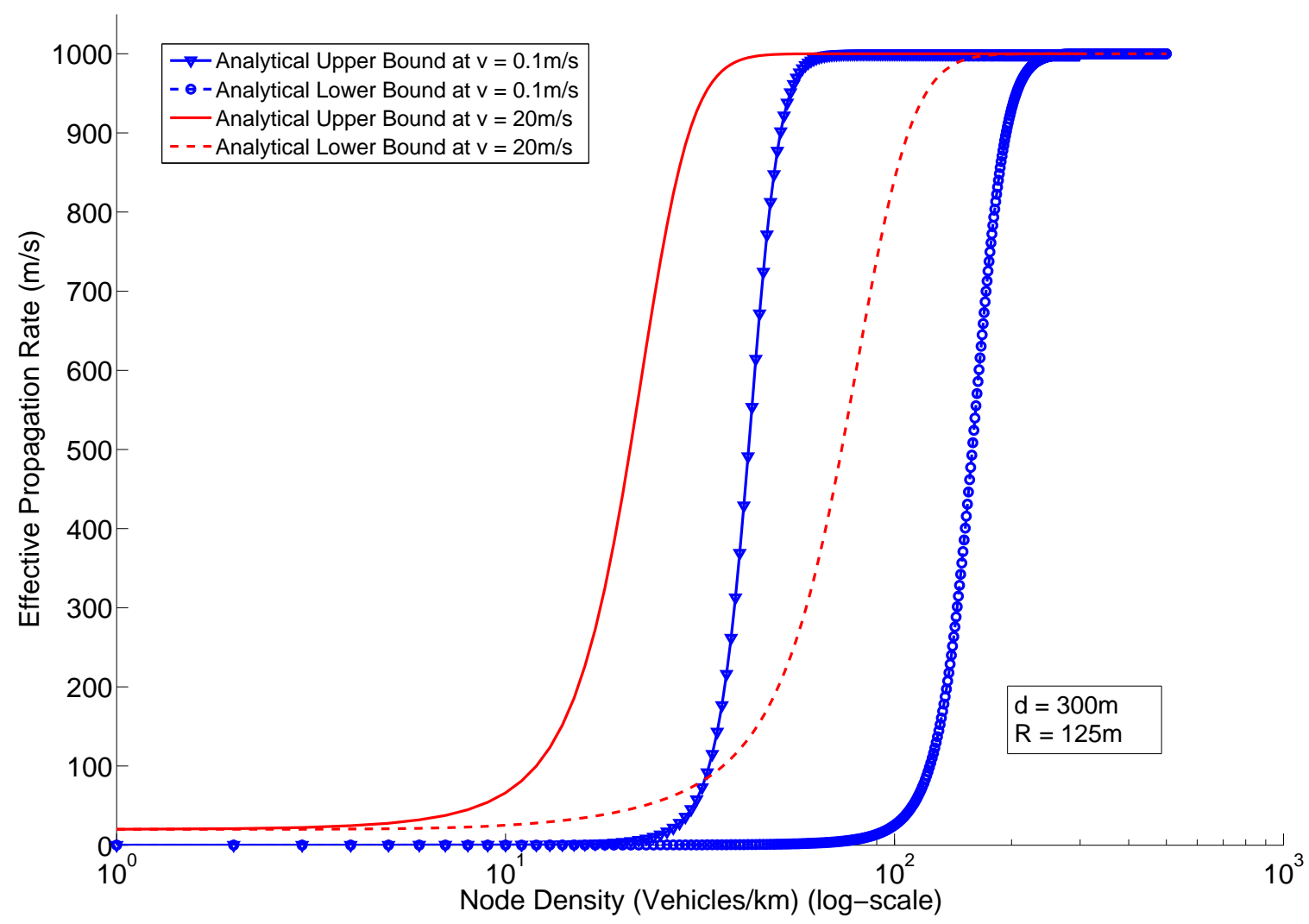

Figure 6: Variation in Effective Propagation Rate at different vehicular speeds.

values of speeds.

Figure 7 shows the analytical and simulation results for the effective propagation rate and its impact on increasing the speed of vehicular traffic. The graph shows an increase in propagation rate with vehicle speed. This result is counter-intuitive - one would expect an increase in traffic speed to create an increased frequency of disconnection, yet it creates increased message propagation due to the rate of topology change. From the simulation results in Figure 7, we observe that an increase in traffic speed from $0 \mathrm{~m} / \mathrm{s}$ to $20 \mathrm{~m} / \mathrm{s}$ results in a corresponding increase in the message propagation rate from $0 \mathrm{~m} / \mathrm{s}$ to at least $200 \mathrm{~m} / \mathrm{s}$. The gain, however, is dependent upon the density of nodes on the highway. A high density of nodes will offer better connectivity and hence, faster propagation.

\section{Summary and Conclusion}

We show an analytical model for characterizing the behavior of message propagation in a VANET routing scheme under varying traffic densities. Simulation studies indicate that the analytical model captures the qualitative behavior of the routing scheme and the impact of vehicular density and vehicular speed on the effective rate of message propagation. The model demonstrates 


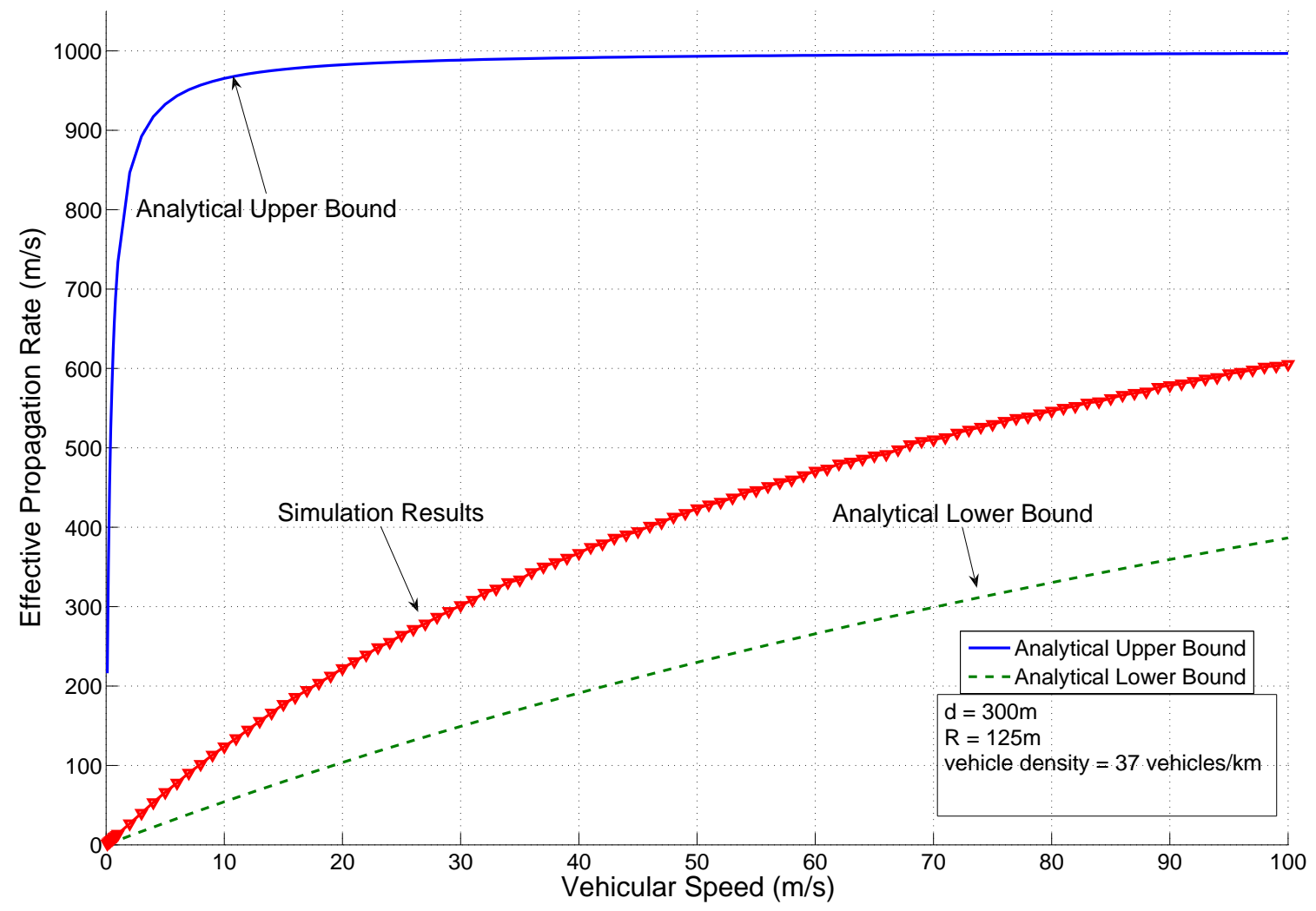

Figure 7: Variation in Effective Propagation Rate with increase in Vehicular Speed (Constant Density).

that the message propagation rate experiences a phase transition behavior as a function of the traffic density. Furthermore, the analysis and simulation demonstrate that opportunistic connections and networking are able to significantly improve message propagation rates at intermediate traffic densities under scenarios in which conventional MANET protocols will fail due to the lack of end-to-end connectivity. An important insight from this work is that it is possible to leverage vehicular mobility for message propagation even when it occurs in the opposing direction. These gains are due to the increased interaction between disconnected fragments in the network. Thus, the results presented in this paper support arguments for the use of a variety of techniques adopted from opportunistic, delay tolerant, mobile ad hoc networking in future vehicular networks.

\section{References}

[1] A. Agarwal and T. D. C. Little, "Prospects for Networked Vehicles of the Future," in Proc. Smart Transportation Workshop in IEEE Real-Time and Embedded Technology and Applications Symposium (RTAS), Bellevue, WA, USA, April 2007.

[2] H. Füßler, M. Mauve, H. Hartenstein, D. Vollmer, and M. Käsemann, "MobiCom poster: Location Based Routing for Vehicular Ad Hoc Networks," in Proc. Intl. Conference on Mobile 
Computing and Networking (MOBICOM '02), vol. 7, no. 1, Atlanta, GA, USA, September 2002, pp. 47-49.

[3] T. D. C. Little and A. Agarwal, "An Information Propagation Scheme for Vehicular Networks," in Proc. IEEE Intelligent Transportation Systems Conference (ITSC), Vienna, Austria, September 2005, pp. 155-160.

[4] I. Berger, "Standards for Car Talk," The Institute, March 2007.

[5] K. I. Farkas and et al., "Vehicular Communication," IEEE Pervasive Computing, vol. 5, no. 4, pp. 55-62, December 2006.

[6] K. Fall, "A Delay-Tolerant Network Architecture for Challenged Internets," in Proc. Special Interest Group on Data Communications (SIGCOMM '03), Karlsruhe, Germany, August 2003, pp. 27-34.

[7] H. Wu, R. Fujimoto, and G. Riley, "Analytical Models for Information Propagation in Vehicle-to-Vehicle Networks," in Proc. 60th IEEE Vehicular Technology Conference (Fall VTC '04), vol. 6, Los Angeles, CA, USA, September 2004, pp. 4548-4552.

[8] V. Naumov, R. Baumann, and T. Gross, "An Evaluation of Inter-Vehicle Ad Hoc Networks Based on Realistic Vehicular Traces," in Proc. 7th ACM Intl. Symp. on Mobile Ad Hoc Networking and Computing (MobiHoc '06), Florence, Italy, May 2006, pp. 108-119.

[9] S. M. Ross, Introduction to Probability Models. Academic Press, 2004, pp. 47-52.

[10] O. Dousse, P. Thiran, and M. Hasler, "Connectivity in Ad-hoc and Hybrid Networks," in Proc. 21st IEEE Conference on Computer Communications (INFOCOM), vol. 2, New York, NY, USA, June 2002, pp. 1079-1088.

[11] H. Wu, R. Fujimoto, R. Guensler, and M. Hunter, "MDDV: A Mobility-Centric Data Dissemination Algorithm for Vehicular Networks," in Proc. 1st ACM Intl. Workshop on Vehicular Ad Hoc Networks (VANET), Philadelphia, PA, USA, 2004, pp. 47-56.

[12] T. Nadeem, P. Shankar, and L. Iftode, "A Comparative Study of Data Dissemination Models for VANETs," in Proc. 3rd Intl. Conference on Mobile and Ubiquitous Systems: Computing, Networking \& Services (MOBIQUITOUS ’06), San Jose, CA, USA, July 2006, pp. 1-10.

[13] J. Burgess, B. Gallagher, D. Jensen, and B. N. Levine, "MaxProp: Routing for Vehicle-Based Disruption-Tolerant Networks," in Proc. IEEE Conference on Computer Communications (INFOCOM), Barcelona, Spain, April 2006, pp. 1-11.

[14] W. Zhao and M. H. Ammar, "Message Ferrying: Proactive Routing in Highly-Partitioned Wireless Ad Hoc Networks," in Proc. 9th IEEE Workshop on Future Trends of Distributed Computing Systems (FTDCS ’03), San Juan, Puerto Rico, 2003, pp. 308-314. 